\title{
Interfaz cerebro-computadora para disminuir situaciones de riesgo en un entorno de conducción vial
}

\author{
Mayra Y. Montiel Sandoval, Pedro C. Santana- \\ Mancilla, Antonio Guerrero Ibáñez
}

Publicado: 21 Septiembre 2016

\begin{abstract}
Resumen
Aplicaciones emergentes que implementan redes de área corporal han marcado una nueva tendencia en la seguridad vehicular. No obstante, la integración de sensores corporales heterogéneos dentro de un entorno de redes vehiculares genera un gran reto, específicamente en la detección de estados de ánimo de los conductores, lo cuales pueden afectar la seguridad vial. Este artículo presenta la propuesta de desarrollo de una arquitectura capaz de integrar una interfaz cerebro-computadora y las redes de comunicación vehicular con el objetivo de recolectar, analizar y clasificar comportamientos de los conductores para identificar situaciones de riesgo en el entorno de conducción y permitir la difusión a tiempo de mensajes de advertencia al resto de conductores dentro de su entorno de conducción contribuyendo a disminuir la probabilidad de que se presente un accidente vial.
\end{abstract}

Palabras clave: Factores humanos; Interfaz Cerebro Computadora; Alertas vehiculares.

\section{Introducción}

En México los accidentes viales representan un grave problema de salud, cada año en promedio mueren 16,500 mexicanos por esta causa. Con base al informe que presenta la Organización Mundial de la Salud, nuestro país ocupa el lugar número trece entre los países que concentran el $62 \%$ del total de fallecimientos por esta causa [1].

La OMS menciona en este informe [7] que la distracción en el tráfico es un factor importante y ha ido en aumento, debido principalmente al uso del teléfono celular y otras tecnologías, afectando el comportamiento de los conductores y reflejado en la disminución de su capacidad cognitiva para reaccionar, aumentando cuatro veces más el riesgo, de verse involucrados en un accidente.

En un esfuerzo por mejorar la seguridad, fiabilidad, eficiencia $\mathrm{y}$ calidad del transporte se han desarrollado un conjunto de soluciones tecnológicas que contribuyan a solventar la problemática mencionada anteriormente. Por un lado se ha creado

\footnotetext{
Montiel Sandoval, MY., Santana-Mancilla, PC., Guerrero Ibáñez, A. Facultad de Telemática Universidad de Colima

Av. Universidad 333, Colima, México

Email:mmontiel@ucol.mx,psantana@ucol.mx,

antonio_guerrero@ucol.mx
}

el concepto de Sistemas Inteligentes de Transporte, que están enfocados a integrar la tecnología al sistema de transporte para crear nuevos servicios, los cuales son empleados para diferentes propósitos y condiciones [5].

La generación de servicios para mejorar la seguridad en los entornos automovilísticos genera gran interés tanto en la industria como en la comunidad científica, evitando accidentes o contribuyendo en la disminución de las consecuencias una vez que estos ya se han producido. Este tipo de tecnologías de comunicaciones dan lugar a redes vehiculares Ad hoc (VANET) en la que los nodos (vehículos) en conjunto con las redes móviles aseguran la disponibilidad de la red para permitir el despliegue de estos servicios. Además las redes móviles han demostrado su eficiencia para el envío de mensajes en amplias áreas geográficas para notificar a múltiples conductores de un peligro concreto de manera extremadamente rápida [11].

Para extender las capacidades de una red VANET se le puede incorporar una red de área personal que permita el uso de sensores ubicados en el cuerpo del conductor para monitorizar signos vitales (ej. señales eléctricas del cerebro, ritmo cardiaco, presión sanguínea, saturación de oxígeno, emociones) o parámetros ambientales (ubicación, temperatura, humedad, iluminación). Comúnmente, estos sensores son estratégicamente colocados sobre el cuerpo humano como pequeños parches u ocultos en prendas permitiendo el monitoreo de signos vitales en tiempo real dentro de su entorno natural por largos períodos de tiempo $[3,4,8]$.

Trabajos previos, han demostrado que el estado emocional del conductor puede generar un nivel de distracción mayor que el uso de teléfonos celulares. Tales emociones pueden causar en los conductores baja capacidad de observación y tiempos reacción, desconocer situaciones como disminución de velocidad abrupta o poder predecir o determinar lo que otros conductores están haciendo o harán. La frustración, ansiedad, miedo y estrés son estados emocionales negativos que pueden afectar la forma de conducir de una persona; por lo que representan una situación de riesgo para sí mismo y para los demás conductores y de ser así podrán ser notificados en sus dispositivos móviles mediante una alarma de manera inmediata y un sistema de geolocalización y difundirlo a través de la red de comunicación hacia otros vehículos que se encuentren en un perímetro cercano y poder tomar la decisión más adecuada.

\section{Planteamiento del problema}

La Organización Mundial de la Salud presenta en su informe sobre la situación de seguridad vial 2013 que los accidentes de 
tránsito siguen mostrando cifras alarmantes cada año. Problemas como congestionamiento vial, largas distancias y tiempo invertido en el traslado afectan directamente a los conductores generando síntomas de estrés, cansancio y como consecuencia una disminución considerable de falta de atención al momento de conducir, lo que puede repercutir en un incremento considerable de la probabilidad de sufrir un accidente [10].

Esta situación demanda la generación de mecanismos que contribuyan a tratar de solventar este problema. Es ahí donde el uso de la tecnología puede ser visualizada como una alternativa viable que complemente y contribuya de manera significativa con las medidas tomadas hasta el momento. Por ello surge la necesidad de crear una solución integral que haga uso de tecnologías emergentes (tantos de comunicación, como de interacción con el usuario) capaz de alertar a los conductores sobre individuos que en su momento representan un riesgo para su seguridad.

\section{Objetivo de la investigación}

El objetivo es utilizar una interfaz cerebro-computadora para monitorizar los estados de ánimo de los conductores, posteriormente analizar y clasificar estos datos para identificar situaciones de riesgo para el conductor y el entorno cercano de conducción; y mediante el uso de un sistema de geolocalización, difundir mensajes de advertencia de la situación al resto de conductores dentro de un área geográfica cercana y de esta manera disminuir la probabilidad de que se presente un accidente vial. La Figura 1 muestra la arquitectura propuesta.
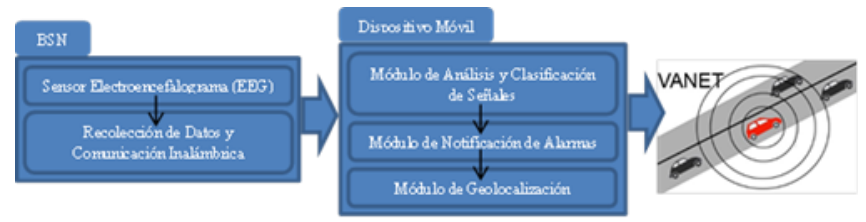

Figura 1. Arquitectura propuesta

Para la obtención de la señal del cerebro, se empleará el sensor Emotiv EPOC que consta de 14 electrodos, los cuales serán colocados según el Sistema Internacional estándar de colocación de electrodos 10-20 y de un dispositivo inalámbrico para la transmisión de las señales obtenidas.

\section{Metodología}

Como se mencionó anteriormente, se propone un escenario en donde el comportamiento del conductor es monitoreado en tiempo real mediante una interfaz cerebro-computadora que detectará las señales eléctricas que produce el cerebro (ondas cerebrales) y el diseño se centrará en usuarios con experiencia en conducción con una edad mínima de 30 años e independiente de su sexo.

\subsection{BSN}

Las señales eléctricas que se recolecten del sensor Emotiv EPOC se enviarán a un dispositivo móvil.

\subsection{Módulo de análisis y clasificación de señales}

El dispositivo móvil contará con una aplicación donde se extraerán las señales alfa $(8-12 \mathrm{~Hz})$ y beta $(12-30 \mathrm{~Hz})$, las cuales serán depuradas eliminando el ruido que contienen con el uso de análisis de frecuencia de Fourier. Dependiendo de la actividad de las frecuencias alfa y beta se activan ciertos estados emocionales [9]. Si la aplicación determina que el conductor está enviando frecuencias que se consideran como un estado emocional que representan un riesgo, tales como frustración, ansiedad, miedo o estrés para sí mismo y para los demás conductores se enviarán al módulo de notificación de alarma.

\subsection{Módulo de notificación de alarmas}

Una vez que se clasifican las frecuencias se seleccionan las que representan una situación de riesgo para los conductores y se envía una un mensaje de alerta. El llevar a cabo la notificación del mensaje significa un nuevo tema de investigación, para que este no represente un distractor para los conductores.

\subsection{Módulo de geolocalización}

Otra aplicación que se requiere en el dispositivo móvil es un sistema de geolocalización para difundir el mensaje de alerta, tanto para el conductor que en ese momento generó la alerta como para los conductores que se encuentren en un perímetro cercano a él.

Para proveer un adecuado soporte a este escenario se propone utilizar el enfoque del diseño centrado en el usuario el cual propone que los diseñadores comprendan el contexto de uso de la aplicación, esto significa llevar una estrecha relación con los usuarios durante todas las fases del desarrollo, las cuales incluyen planificación, diseño, desarrollo y evaluación [2].

\section{Conclusiones}

Este trabajo propone una arquitectura capaz de integrar redes inalámbricas de área corporal en una red vehicular ad hoc para la difusión de mensajes de alerta de conductores que representan un riesgo según su estado emocional a través de una red móvil, permitiendo que exista una interacción entre los conductores y su dispositivo móvil de manera eficiente y segura, ofreciendo la oportunidad de tomar una decisión oportuna ante una situación que represente una riesgo para su salud.

\section{Referencias}

[1] CONAPRA. Tercer informe sobre la situación de la seguridad vial, México 2013. Consejo Nacional para la Prevención de Accidentes, 2013.

[2] Gulliksen, J., Lantz, A., and Boivie, I. User Centered Design in Practice - Problems and Possibilities. Royal Institute of Technology, Stockhom, Sweden, 1999.

[3] Horrey, W.J. and Wickens, C.D. Examining the impact of cell phone conversations on driving using meta-analytic techniques. Human Factors 48, 1 (2006), 196-205.

[4] Kojima, S., Maeda, S., Ogura, Y., et al. Noninvasive biological sensor system for detection of drunk driving. IEEE (2009), 1-4.

[5] Nandgaonkar, S., Parulekar, G., and Desai, D. Intelligent Transport Systems And Road Safety - A Survey. IOSR Journal of C ivil Engineering (IOSR - J M CE), (2009), 4345.

[6] OMS. Informe sobre la situación mundial de la seguridad vial 2015. Organización Mundial de la Salud, 2015.

[7] OMS and NHTSA. Uso del celular al volante: un problema creciente de distracción del conductor. Organización Mundial de la Salud, Suiza, 2011.

[8] Otto, C., Milenkovic, A., Sanders, C., and Jovanov, E. System architecture of a wireless body area sensor network for ubiquitous health monitoring. Journal of Mobile Multimedia 1, 4 (2005), 307-326. 
[9] Ramirez, R. and Vamvakousis, Z. Detecting Emotion from EEG Signals Using the Emotive Epoc Device. In F.M.

Zanzotto, S. Tsumoto, N. Taatgen and Y. Yao, eds., Brain Informatics. Springer Berlin Heidelberg, Berlin, Heidelberg, 2012, 175-184.

[10] Rigas, G., Goletsis, Y., and Fotiadis, D.I. Real-Time Driver's Stress Event Detection. IEEE Transactions on Intelligent Transportation Systems 13, 1 (2012), 221-234.
[11] Yungpeng, Z., Sories S, Gehlen Guido, and Walke Bernhard. Towards a European Solution for Networked Cars -

Integration of Car-to-Car technology into cellular systems for vehicular communication in Europe. (2009). 